\title{
A SEPARATED COHOMOLOGICALLY COMPLETE MODULE IS COMPLETE
}

\author{
AMNON YEKUTIELI
}

\begin{abstract}
We prove two theorems on cohomologically complete complexes. These theorems are inspired by, and yield an alternative proof of, a recent theorem of P. Schenzel on complete modules.
\end{abstract}

Throughout $A$ denotes a nonzero commutative ring. (We do not assume $A$ is noetherian.) The category of $A$-modules is denoted by $\operatorname{Mod} A$, its category of unbounded complexes is $\mathrm{C}(\operatorname{Mod} A)$, and the derived category is $\mathrm{D}(\operatorname{Mod} A)$.

Let $\mathfrak{a}$ be a finitely generated ideal in $A$. Recall that the $\mathfrak{a}$-adic completion of an $A$-module $M$ is

$$
\Lambda_{\mathfrak{a}}(M):=\lim _{\leftarrow k}\left(\left(A / \mathfrak{a}^{k}\right) \otimes_{A} M\right) .
$$

The module $M$ is called $\mathfrak{a}$-adically complete (resp. a-adically separated) if the canonical homomorphism $\tau_{M}: M \rightarrow \Lambda_{\mathfrak{a}}(M)$ is bijective (resp. injective). It is known that the completion $\Lambda_{\mathfrak{a}}(M)$ is $\mathfrak{a}$-adically complete (see [Ye Corollary 3.6]).

The additive functor

$$
\Lambda_{\mathfrak{a}}: \operatorname{Mod} A \rightarrow \operatorname{Mod} A
$$

has a left derived functor

$$
\mathrm{L} \Lambda_{\mathfrak{a}}: \mathrm{D}(\operatorname{Mod} A) \rightarrow \mathrm{D}(\operatorname{Mod} A) .
$$

A complex $M \in \mathrm{D}(\operatorname{Mod} A)$ is called cohomologically $\mathfrak{a}$-adically complete if the canonical morphism $\tau_{M}^{\mathrm{L}}: M \rightarrow \mathrm{L} \Lambda_{\mathfrak{a}}(M)$ in $\mathrm{D}(\operatorname{Mod} A)$ is an isomorphism. We say that an $A$-module $M$ is cohomologically complete if it is so as a complex, using the standard embedding $\operatorname{Mod} A \rightarrow \mathrm{D}(\operatorname{Mod} A)$. See [PSY1] for more details.

The next example (taken from [Ye]) shows an anomalous module $M$ : it is cohomologically a-adically complete, but not $\mathfrak{a}$-adically complete.

First we have to recall some concepts from [Ye]. Assume $A$ is a-adically complete, and let $Z$ be some set. The module of finitely supported functions $f: Z \rightarrow A$, denoted by $\mathrm{F}_{\text {fin }}(Z, A)$, is free with basis the collection $\left\{\delta_{z}\right\}_{z \in Z}$ of delta functions. Its $\mathfrak{a}$-adic completion is canonically isomorphic to the module $\mathrm{F}_{\operatorname{dec}}(Z, A)$ of $\mathfrak{a}$ adically decaying functions; see [Ye, Definition 2.1 and Corollary 2.9]. An $A$-module $P$ is called $\mathfrak{a}$-adically free if it is isomorphic to $\mathrm{F}_{\mathrm{dec}}(Z, A)$ for some set $Z$.

Example 1. Let $A:=\mathbb{K}[[t]]$, the power series ring in a variable $t$ over a field $\mathbb{K}$, and $\mathfrak{a}:=(t)$. Define the $\mathfrak{a}$-adically free $A$-modules $P^{0}=P^{-1}:=\mathrm{F}_{\operatorname{dec}}(\mathbb{N}, A)$. Let

Date: 25 April 2014 bis.

Key words and phrases. adic completion, derived completion.

Mathematics Subject Classification 2010. Primary: 13B35; Secondary: 18G10, 13J10. 
$\mathrm{d}: P^{-1} \rightarrow P^{0}$ be the homomorphism $\mathrm{d}\left(\delta_{i}\right):=t^{i} \delta_{i}$. Define $P:=\left(P^{-1} \stackrel{\mathrm{d}}{\rightarrow} P^{0}\right)$, a complex concentrated in degrees $-1,0$; and let $M:=\mathrm{H}^{0}(P) \in \operatorname{Mod} A$.

Consider the canonical surjection $\pi^{0}: P^{0} \rightarrow M$, and $m:=\pi^{0}\left(\sum_{i \in \mathbb{N}} t^{i} \delta_{i}\right) \in M$. The element $m$ is nonzero, but it belongs to $\cap_{i \in \mathbb{N}} \mathfrak{a}^{i} M$. Therefore $M$ is not an $\mathfrak{a}$ adically separated module, and hence it is not an a-adically complete module. (This was already noticed in [Si2, Example 2.5].)

On the other hand, the canonical homomorphism $\pi: P \rightarrow M$ is a quasiisomorphism, so according to [PSY2, Theorem 1.15] the module $M$ is cohomologically a-adically complete.

Remark 2. Here are a few words regarding the history and background. The total left derived functor $\mathrm{L} \Lambda_{\mathfrak{a}}$ was first studied in $[\mathrm{AJL}]$, following earlier work on the left derived functors $\mathrm{L}_{i} \Lambda_{\mathfrak{a}}=\mathrm{H}^{-i}\left(\mathrm{~L} \Lambda_{\mathfrak{a}}\right)$, mostly in [GM]. See also [Ma] and [Si1].

Many important properties of $\mathrm{L} \Lambda_{\mathfrak{a}}$ can be found in the paper [AJL]. However, we prefer to quote [PSY1, PSY2], where the relevant theory was developed further.

The definition of cohomologically complete complexes above was introduced in [PSY1]. The name actually originates in [KS], but the definition there is different (yet equivalent, as proved in [PSY1, Theorem 1.4]).

Recently we came across a remarkable new result of P. Schenzel ([SC, Theorem 1.1], which is the noetherian case of Theorem 10 below). While trying to understand this result, we discovered the next theorem, which explains the anomaly of the module $M$ in Example 1

Let us denote by $\mathrm{D}^{\mathrm{b}}(\operatorname{Mod} A)$ the full subcategory of $\mathrm{D}(\operatorname{Mod} A)$ consisting of complexes with bounded cohomologies.

Theorem 3. Let $A$ be a noetherian commutative ring, let $\mathfrak{a}$ be an ideal in $A$, and let $M \in \mathrm{D}^{\mathrm{b}}(\operatorname{Mod} A)$. The following conditions are equivalent:

(i) The A-modules $\mathrm{H}^{j}(M)$ are $\mathfrak{a}$-adically complete for all $j \in \mathbb{Z}$.

(ii) The complex $M$ is a-adically cohomologically complete, and the A-modules $\mathrm{H}^{j}(M)$ are $\mathfrak{a}$-adically separated for all $j \in \mathbb{Z}$.

Proof. (i) $\Rightarrow$ (ii): This is immediate from [PSY2, Theorem 1.21].

(ii) $\Rightarrow$ (i): Here we have to do some work. Recall that for a graded $A$-module $N=\bigoplus_{i \in \mathbb{Z}} N^{i}$ its amplitude is

$$
\operatorname{amp}(N):=\sup (N)-\inf (N) \in \mathbb{Z} \cup\{ \pm \infty\} .
$$

The amplitude satisfies $\operatorname{amp}(N)=-\infty$ iff $N=0$, and $\operatorname{amp}(N)<\infty$ iff $N$ is bounded. Cf. [PSY1, formulas (2.3)-(2.5)]. We proceed by induction on $\operatorname{amp}(\mathrm{H}(M))$.

If $M=0$ there is nothing to prove. So let us assume that $0 \leq \operatorname{amp}(\mathrm{H}(M))<$ $\infty$. Let $j:=\sup (\mathrm{H}(M))$. According to [PSY2, Theorem 1.15], there a quasiisomorphism $P \rightarrow M$, where $P$ is a complex of a-adically free modules, and $\sup (P)=j$. Let $\pi: P^{j} \rightarrow \mathrm{H}^{j}(P)$ be the canonical surjection. Since $\tau: \mathbf{1} \rightarrow \Lambda_{\mathfrak{a}}$ is a 
natural transformation, there is a commutative diagram

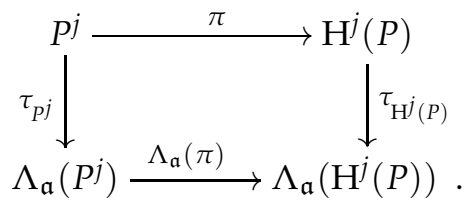

We know that the functor $\Lambda_{\mathfrak{a}}$ preserves surjections, so $\Lambda_{\mathfrak{a}}(\pi)$ is also surjective. Since $P^{j}$ is complete, the homomorphism $\tau_{P^{j}}$ is bijective. Therefore $\tau_{\mathrm{H}^{j}(P)}$ is surjective. On the other hand, by assumption the module $\mathrm{H}^{j}(P) \cong \mathrm{H}^{j}(M)$ is separated, so the homomorphism $\tau_{\mathrm{H}^{j}(P)}$ is injective. Thus $\tau_{\mathrm{H}^{j}(P)}$ is bijective, and we conclude that $\mathrm{H}^{j}(M)$ is a-adically complete.

Using smart truncation of $M$ at $j$ there is a distinguished triangle

$$
M^{\prime} \rightarrow M \rightarrow \mathrm{H}^{j}(M)[-j] \rightarrow M^{\prime}[1]
$$

in $\mathrm{D}(\operatorname{Mod} A)$, such that $\mathrm{H}^{j^{\prime}}\left(M^{\prime}\right) \cong \mathrm{H}^{j^{\prime}}(M)$ for all $j^{\prime}<j$, and $\mathrm{H}^{j^{\prime}}\left(M^{\prime}\right)=0$ for all $j^{\prime} \geq j$. By the implication (i) $\Rightarrow$ (ii) we know that the module $\mathrm{H}^{j}(M)$ is cohomologically complete. Since the category $\mathrm{D}(\operatorname{Mod} A)_{\mathfrak{a} \text {-com }}$ is triangulated, it follows that $M^{\prime}$ is also cohomologically complete. $\operatorname{But} \operatorname{amp}\left(\mathrm{H}\left(M^{\prime}\right)\right)<\operatorname{amp}(\mathrm{H}(M))$, so induction tells us that the modules $\mathrm{H}^{j^{\prime}}\left(M^{\prime}\right)$ are all complete.

Remark 4. We do not know whether Theorem 3 holds without assuming that $\mathrm{H}(M)$ is bounded. Perhaps ideas in [Si1] can shed some light on this question.

Here is our second new result on cohomologically complete complexes.

Theorem 5. Let $A$ be a noetherian commutative ring, let $\mathfrak{a}_{1}, \ldots, \mathfrak{a}_{n}$ be ideals in $A$, let $\mathfrak{a}:=\mathfrak{a}_{1}+\cdots+\mathfrak{a}_{n}$, and let $M \in \mathrm{D}(\operatorname{Mod} A)$. The following conditions are equivalent:

(i) $M$ is a-adically cohomologically complete.

(ii) $M$ is $\mathfrak{a}_{i}$-adically cohomologically complete for all $i=1, \ldots, n$.

Proof. For every $i$ choose a finite sequence $\boldsymbol{a}_{i}$ that generates the ideal $\mathfrak{a}_{i}$. Let $\boldsymbol{a}:=$ $\left(a_{1}, \ldots, a_{n}\right)$, the concatenated sequence, and let $\boldsymbol{b}_{i}$ be the sequence gotten from $\boldsymbol{a}$ be deleting $\boldsymbol{a}_{i}$. Define $T:=\operatorname{Tel}(A ; \boldsymbol{a}), T_{i}:=\operatorname{Tel}\left(A ; \boldsymbol{a}_{i}\right)$ and $S_{i}:=\operatorname{Tel}\left(A ; \boldsymbol{b}_{i}\right)$, the telescope complexes from [PSY1, Definition 5.1]. Note that

$$
T \cong T_{1} \otimes_{A} \cdots \otimes_{A} T_{n} \cong T_{i} \otimes_{A} S_{i}
$$

in $C(\operatorname{Mod} A)$.

There is a canonical homomorphism $u: T \rightarrow A$ in C $(\operatorname{Mod} A)$, see [PSY1, formula 5.6]. According to [PSY1, Corollary 5.25], $M$ is a-adically cohomologically complete iff the homomorphism

$$
\operatorname{Hom}\left(u, \mathbf{1}_{M}\right): M \rightarrow \operatorname{Hom}_{A}(T, M)
$$

in $\mathrm{C}(\operatorname{Mod} A)$ is a quasi-isomorphism. Likewise there are homomorphisms $u_{i}$ : $T_{i} \rightarrow A$, and $M$ is $\mathfrak{a}_{i}$-adically cohomologically complete iff the homomorphism

$$
\operatorname{Hom}\left(u_{i}, \mathbf{1}_{M}\right): M \rightarrow \operatorname{Hom}_{A}\left(T_{i}, M\right)
$$

is a quasi-isomorphism. 
Let us prove the implication (i) $\Rightarrow$ (ii). For every index $i$ there is a diagram

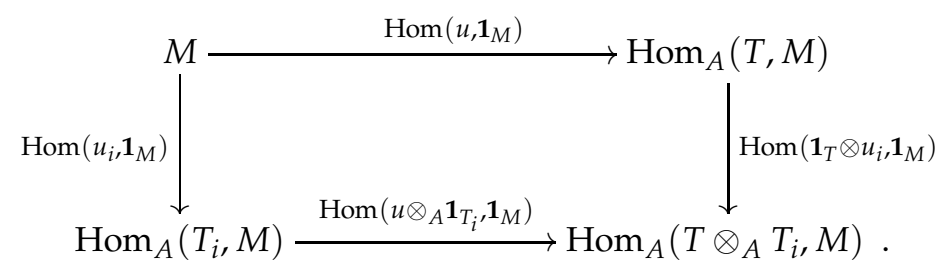

in $\mathrm{C}(\operatorname{Mod} A)$, which is commutative up to sign. By assumption the homomorphism $\operatorname{Hom}\left(u, \mathbf{1}_{M}\right)$ is a quasi-isomorphism. By adjunction there is an isomorphism

in $\mathrm{C}(\operatorname{Mod} A)$, and it sends

$$
\operatorname{Hom}_{A}\left(T \otimes_{A} T_{i}, M\right) \cong \operatorname{Hom}_{A}\left(T_{i}, \operatorname{Hom}_{A}(T, M)\right)
$$

$$
\operatorname{Hom}\left(u \otimes_{A} \mathbf{1}_{T_{i}}, \mathbf{1}_{M}\right) \mapsto \pm \operatorname{Hom}\left(\mathbf{1}_{T_{i}}, \operatorname{Hom}_{A}\left(u, \mathbf{1}_{M}\right)\right) .
$$

Because $T_{i}$ is K-projective, we see that $\operatorname{Hom}\left(u \otimes_{A} \mathbf{1}_{T_{i}}, \mathbf{1}_{M}\right)$ is a quasi-isomorphism.

By [PSY1, Lemma 7.9] the homomorphism $u_{i}: T_{i} \otimes_{A} T_{i} \rightarrow T_{i}$ is a homotopy equivalence. This implies that

$$
\mathbf{1}_{T} \otimes u_{i}= \pm \mathbf{1}_{T_{i}} \otimes \mathbf{1}_{S_{i}} \otimes u_{i}: S_{i} \otimes_{A} T_{i} \otimes_{A} T_{i} \rightarrow S_{i} \otimes_{A} T_{i}
$$

is a homotopy equivalence, and therefore $\operatorname{Hom}\left(\mathbf{1}_{T} \otimes u_{i}, \mathbf{1}_{M}\right)$ is a quasi-isomorphism. The conclusion is that $\operatorname{Hom}\left(u_{i}, \mathbf{1}_{M}\right)$ is a quasi-isomorphism.

Finally we prove that (ii) $\Rightarrow$ (i). The assumption is that $M \rightarrow \operatorname{Hom}_{A}\left(T_{i}, M\right)$ are quasi-isomorphisms. Applying $\operatorname{Hom}_{A}\left(T_{2},-\right)$ to the quasi-isomorphism $M \rightarrow$ $\operatorname{Hom}_{A}\left(T_{1}, M\right)$, we get a quasi-isomorphism

$$
\operatorname{Hom}_{A}\left(T_{2}, M\right) \rightarrow \operatorname{Hom}_{A}\left(T_{2}, \operatorname{Hom}_{A}\left(T_{1}, M\right)\right) \text {. }
$$

Continuing this way we end up with a sequence of quasi-isomorphisms

$$
M \rightarrow \operatorname{Hom}_{A}\left(T_{n}, M\right) \rightarrow \cdots \rightarrow \operatorname{Hom}_{A}\left(T_{n}, \cdots, \operatorname{Hom}_{A}\left(T_{1}, M\right) \cdots\right) .
$$

Using adjunction we get an isomorphism

$$
\operatorname{Hom}_{A}\left(T_{n}, \cdots, \operatorname{Hom}_{A}\left(T_{1}, M\right) \cdots\right) \cong \operatorname{Hom}_{A}\left(T_{1} \otimes_{A} \cdots \otimes_{A} T_{n}, M\right)
$$

in $\mathrm{C}(\operatorname{Mod} A)$. Up to sign, the resulting quasi-isomorphism $M \rightarrow \operatorname{Hom}_{A}(T, M)$ is $\operatorname{Hom}\left(u, \mathbf{1}_{M}\right)$.

Remark 6. In Theorems 3 and 5 we can remove the assumption that the ring $A$ is noetherian, and replace it by the weaker assumption that the ideals $\mathfrak{a}, \mathfrak{a}_{1}, \ldots, \mathfrak{a}_{n}$ are weakly proregular; see [PSY1]. This observation was communicated to us by L. Shaul.

Lemma 7. Assume $A$ is noetherian, and $\mathfrak{a}$ is a principal ideal, generated by an element $a$. Let $M$ be an A-module.

(1) The following conditions are equivalent:

(i) $M$ is a-adically cohomologically complete.

(ii) $\operatorname{Ext}_{A}^{j}\left(A_{a}, M\right)=0$ for $j=0,1$.

(2) If $M$ is a-adically separated, then $\operatorname{Ext}_{A}^{0}\left(A_{a}, M\right)=0$.

In the lemma, $A_{a}$ is the localization of $A$ w.r.t. the element $a$. 
Proof. (1) In the principal case the DG algebra $\mathrm{C}(A ; \boldsymbol{a})$ appearing in [PSY1, Section 8] is just the localized ring $A_{a}$. Thus by [PSY1, Theorem 8.8], $M$ is a-adically cohomologically complete iff $\operatorname{RHom}_{A}\left(A_{a}, M\right)=0$.

The telescope complex $\operatorname{Tel}(A ; a)$ from [PSY1, Definition 5.1] is a complex of free $A$-modules

$$
\operatorname{Tel}(A ; a)=\left(\cdots \rightarrow 0 \rightarrow \mathrm{F}_{\text {fin }}(\mathbb{N}, A) \rightarrow \mathrm{F}_{\text {fin }}(\mathbb{N}, A) \rightarrow 0 \rightarrow \cdots\right)
$$

concentrated in degrees 0,1 . The infinite dual Koszul complex

$$
\mathrm{K}_{\infty}^{\vee}(A ; a)=\left(\cdots \rightarrow 0 \rightarrow A \rightarrow A_{a} \rightarrow 0 \rightarrow \cdots\right)
$$

is a complex of flat $A$-modules, also concentrated in degrees 0,1 . According to [PSY1, Lemma 5.7] there is a quasi-isomorphism

$$
w_{a}: \operatorname{Tel}(A ; a) \rightarrow \mathrm{K}_{\infty}^{\vee}(A ; a) .
$$

Looking at the definitions of $\operatorname{Tel}(A ; a)$ and $w_{a}$, we see that passing to the subcomplex

$$
\operatorname{Tel}_{+}(A ; a):=\left(\cdots \rightarrow 0 \rightarrow \mathrm{F}_{\text {fin }}([1, \infty], A) \rightarrow \mathrm{F}_{\text {fin }}(\mathbb{N}, A) \rightarrow 0 \rightarrow \cdots\right)
$$

of $\operatorname{Tel}(A ; a)$, i.e. omitting the module $A \delta_{0}$ in degree 0 , we get an induced a quasiisomorphism

$$
w_{a}: \operatorname{Tel}_{+}(A ; a) \rightarrow A_{a}[-1] .
$$

Therefore

$$
\operatorname{RHom}_{A}\left(A_{a}, M\right) \cong \operatorname{Hom}_{A}\left(\operatorname{Tel}_{+}(A ; a)[1], M\right)
$$

has cohomology only in degrees 0,1 .

(2) Assume $M$ is a-adically separated, and let $\widehat{M}:=\Lambda_{\mathfrak{a}}(M)$. Since $\tau_{M}: M \rightarrow \widehat{M}$ is injective, we have an injection $\operatorname{Hom}_{A}\left(A_{a}, M\right) \rightarrow \operatorname{Hom}_{A}\left(A_{a}, \widehat{M}\right)$. But $\widehat{M}$ is $\mathfrak{a}$ adically complete, so by Theorem 3 it is a-adically cohomologically complete, and thus by part (1) we know that $\operatorname{Hom}_{A}\left(A_{a}, \widehat{M}\right)=0$.

Lemma 8. Suppose $f: A \rightarrow B$ is a ring homomorphism, $a \in A, b:=f(a) \in B$, and $M \in \operatorname{Mod} B$. For every $i$ there is a canonical B-module isomorphism

$$
\operatorname{Ext}_{B}^{i}\left(B_{b}, M\right) \cong \operatorname{Ext}_{A}^{i}\left(A_{a}, M\right) \text {. }
$$

Proof. There is an isomorphism of complexes of $B$-modules

$$
B \otimes_{A} \operatorname{Tel}_{+}(A ; a) \cong \mathrm{Tel}_{+}(B ; b) .
$$

This induces an isomorphism

$$
\begin{aligned}
& \operatorname{Ext}_{B}^{i}\left(B_{b}, M\right) \cong \mathrm{H}^{i}\left(\operatorname{Hom}_{B}\left(\operatorname{Tel}_{+}(B ; b)[1], M\right)\right) \\
& \quad \cong \mathrm{H}^{i}\left(\operatorname{Hom}_{A}\left(\operatorname{Tel}_{+}(A ; a)[1], M\right)\right) \cong \operatorname{Ext}_{A}^{i}\left(A_{a}, M\right) .
\end{aligned}
$$

Lemma 9. Let $\mathfrak{b} \subset \mathfrak{a}$. If an $A$-module $M$ is $\mathfrak{a}$-adically separated, then it is also $\mathfrak{b}$-adically separated.

Proof. Since $\mathfrak{b}^{i} M \subset \mathfrak{a}^{i} M$ for all $i$, we have $\bigcap_{i} \mathfrak{b}^{i} M \subset \bigcap_{i} \mathfrak{a}^{i} M=0$.

Schenzel's $[\mathrm{Sc}$. Theorem 1.1] is the next theorem, when $A$ is noetherian. This condition turns out to be unnecessary. 
Theorem 10. Let $A$ be a commutative ring, let $\mathfrak{a}$ be a finitely generated ideal in $A$, and let $\left(a_{1}, \ldots, a_{n}\right)$ be a sequence of elements that generates $\mathfrak{a}$. The following conditions are equivalent for any A-module $M$ :

(i) $M$ is a-adically complete.

(ii) $M$ is a-adically separated, and $\operatorname{Ext}_{A}^{1}\left(A_{a_{i}}, M\right)=0$ for all $i=1, \ldots, n$.

Proof. Step 1. Assume $A$ is noetherian. For any $i$ let $\mathfrak{a}_{i}$ be the ideal generated by the elements $a_{i}$. Consider these further conditions:

(n1) $M$ is a-adically separated and a-adically cohomologically complete.

(n2) $M$ is a-adically separated, and $\mathfrak{a}_{i}$-adically cohomologically complete for all $i=1, \ldots, n$.

(n3) $M$ is $\mathfrak{a}$-adically separated, and $\mathfrak{a}_{i}$-adically complete for all $i=1, \ldots, n$.

By Theorem 3 we have (i) $\Leftrightarrow(\mathrm{n} 1)$. By Theorem 5 we have $(\mathrm{n} 1) \Leftrightarrow(\mathrm{n} 2)$. Combining Lemma 9 and Theorem 3 we deduce the equivalence $(\mathrm{n} 2) \Leftrightarrow(\mathrm{n} 3)$. Finally, the equivalence (n3) $\Leftrightarrow$ (ii) comes from the combination of Lemmas 9 and 7

Step 2. Now $A$ is arbitrary. Consider the polynomial ring $\mathbb{Z}[\boldsymbol{t}]:=\mathbb{Z}\left[t_{1}, \ldots, t_{n}\right]$, the ideal $\mathfrak{t}:=\left(t_{1}, \ldots, t_{n}\right)$, and the ring homomorphism $f: \mathbb{Z}[\boldsymbol{t}] \rightarrow A, f\left(t_{i}\right):=a_{i}$. Since $\mathfrak{a}^{k} M=\mathfrak{t}^{k} M$ for every $k \in \mathbb{N}$, we see that $M$ is a-adically complete (resp. separated) iff it is t-adically complete (resp. separated). On the other hand, by Lemma 8 we know that $\operatorname{Ext}_{A}^{1}\left(A_{a_{i}}, M\right)=0$ iff $\operatorname{Ext}_{\mathbb{Z}[t]}^{1}\left(\mathbb{Z}[\boldsymbol{t}]_{t_{i}}, M\right)=0$. Since the ring $\mathbb{Z}[\boldsymbol{t}]$ is noetherian, we are done by step 1 .

Acknowledgments. I wish to thank Liran Shaul for helpful discussions. Thanks also to the anonymous referee for a careful reading of the paper and for several suggestions.

\section{REFERENCES}

[AJL] L. Alonso, A. Jeremias and J. Lipman, Local homology and cohomology on schemes, Ann. Sci. ENS 30 (1997), 1-39. Correction, availabe online at http://www.math.purdue.edu/ lipman/papers/homologyfix.pdf

[GM] J.P.C. Greenlees and J.P. May, Derived functors of I-adic completion and local homology, J. Algebra 149 (1992), 438-453.

[KS] M. Kashiwara and P. Schapira, Astérisque, Soc. Math. France, 345 (2012)

[Ma] E. Matlis, The Higher Properties of R-Sequences, J. Algebra 50 (1978), 77-112.

[PSY1] M. Porta, L. Shaul and A. Yekutieli, On the homology of completion and torsion, Algebras and Representation Theory 17 (2014), 31-67. Online http://dx.doi.org/10.1007/s10468-012-9385-8

[PSY2] M. Porta, L. Shaul and A. Yekutieli, Cohomologically Cofinite Complexes, to appear in Communications in Algebra, online http://arxiv.org/abs/1208.4064

[Sc] P. Schenzel, A criterion for I-Adic completeness, Arch. Math. 102 (2014), 25-33.

[Si1] A.M. Simon, Some homological properties of complete modules, Math. Proc. Camb. Phil. Soc. 108 (1990), 231-246.

[Si2] A.M. Simon, Approximations of Complete Modules by Complete Big Cohen-Macaulay Modules over a Cohen-Macaulay Local Ring, Algebr. Represent. Theor. 12 (2009), 385-400.

[Ye] A. Yekutieli, On Flatness and Completion for Infinitely Generated Modules over Noetherian Rings, Communications in Algebra 39, Issue 11 (2011), 4221-4245.

Department of Mathematics, Ben GURion University, Be'ER SHeVA 84105, IsRael

E-mail address: amyekut@math.bgu.ac.il 